\title{
Detección de Brucella canis por método de inmunocromatografía en perros vagos capturados en la ciudad de Temuco, Chile, 2011
}

\author{
Christian Tuemmers, Carlos Lüders, Claudio Rojas, Michel Serri, Carolina Castillo y Rodrigo Espinoza
}

\section{Detection of Brucella canis by immunochromatography method in vague dogs captured in Temuco city, Chile, 2011}

Background: Brucella canis is responsible for brucellosis in dogs, causing reproductive disorders and is considered a zoonoses, as described in several countries. The epidemiological data are scarce in our country. Aim: To determine the prevalence of Brucella canis in vague dogs in Temuco city and housed in the Temuco Kennel. Methods: Quantitative and cross-section study. We used 400 samples of dogs of both sexes, different ages and mainly mixed race, which were tested by immunochromatography. Results: Antibodies were detected in 4 samples Brucella canis which represented $1 \%$ of the population studied, 2 females $(0.5 \%)$ and 2 males $(0.5 \%)$. Discussion: We conclude that dogs are infected by B. canis in a low range but remains a risk condition to the health of the human population if not maintained adequate sanitary control of pets, like vague dogs.

Key words: Brucella canis, dog, zoonoses.

Palabras clave: Brucella canis, perro, zoonosis.

\section{Introducción}

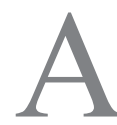

ctualmente se reconocen nueve especies en el género Brucella: $B$. melitensis, es la especie más importante por ser altamente nociva, posee tres biotipos y afecta principalmente a caprinos y ovinos; $B$. ovis, llamada así porque sólo infecta ovinos; B. abortus, que tiene siete biotipos y es capaz de propagarse en bovinos, búfalos y visón; $B$. suis, que tiene una amplia especificidad (liebres, reno, caribú y roedores) puesto que tiene 5 biovares -sin embargo, su principal hospedero es el porcino-; $B$. canis, es principalmente patógena en caninos; $B$. neotomae, que es especie específica de roedo$\operatorname{res}^{1-3}, B$. microti, se le atribuye la enfermedad en el zorro rojo y ratón de campo ${ }^{4}, B$. ceti, que se puede transmitir a cetáceos (ballenas, marsopas y delfines) y, por último, $B$. pinnipedialis, para las cepas de pinnípedos como focas, elefantes marinos y morsas ${ }^{2,5}$.

De las nueve especies, cinco pueden ser patógenas para el ser humano. Basándose en su patogenicidad, el orden ascendente en que se ubican es: $B$. canis, B. abortus, $B$. suis y $B$. melitensis, siendo esta última la más agresiva ${ }^{6}$. También se han documentado casos donde B. maris ha demostrado ser una zoonosis ${ }^{2}$; sin embargo, por ser recientemente descubierta no se conocen datos más relevantes.

La brucelosis, causada específicamente por $B$. $c a$ nis, es una zoonosis poco frecuente. Existe un amplio espectro de signos y síntomas detectados en pacientes que padecen brucelosis causada por B. canis, lo que dificulta el diagnóstico de esta patología ${ }^{7,8}$. Cuando nos enfrentamos a un paciente febril con signos y síntomas de origen desconocido y antecedentes de contacto con perros, debe ser considerada la brucelosis como un pre diagnóstico. En este tipo de pacientes podemos encontrar signos tan variables e inespecíficos como fiebre de origen desconocido, debilidad, disminución de la condición corporal, linfoadenopatía generalizada, visceromegalia, atrofia muscular e incluso disnea, ortopnea y edema de miembros inferiores producto de una endocarditis infecciosa ocasionada por B. canis. Esta localización es la principal causa de mortalidad por esta patología, pues en $82 \%$ de los casos se ve afectada principalmente la válvula aórtica. Existe poca información en la literatura médica sobre el curso clínico de la brucelosis en pacientes afectados por otras enfermedades, aunque se ha comunicado en pacientes con infección por $\mathrm{VIH}^{9}$.

En perros la signología es variable e inespecífica; se pueden encontrar signos tales como letargo, pérdida de peso y condición corporal, intolerancia al ejercicio, apatía, disminución o pérdida de la libido, envejecimiento prematuro, pelaje hirsuto y lo más común de esta enfermedad: linfoadenitis generalizada ${ }^{10,11}$; o, por el contrario, puede presentarse de forma asintomática durante todo el transcurso de la infección. En el caso de afectar de forma específica a algún órgano en particular, puede cursar de manera aguda comprometiendo principalmente al sistema
Universidad Católica de Temuco, Chile.

Financiamiento: Proyecto FONIS SA10120029

Conflictos de interés: ninguno

Recibido: 30 de octubre de 2012 Aceptado: 10 de marzo de 2013

Correspondencia a:

Christian Tuemmers

ctuemmers@uct.cl 
reproductivo (aborto, atrofia testicular, epididimitis, prostatitis), el tejido retículo endotelial (hepatomegalia, esplenomegalia) y el sistema osteoarticular (discoespondilitis, artritis, artralgia) ${ }^{10-12}$.También se puede presentar de forma crónica debido a la capacidad de $B$. canis de evadir los mecanismos de defensa del hospedero y permanecer como microorganismo intracelular en los tejidos, por un largo tiempo ${ }^{14,15}$

Debido a su potencial zoonótico, B. canis ha sido motivo de estudio para muchos países. Su prevalencia es variable según la región, con descripción de casos desde su primer aislamiento en el año 1966. Shin y Carmichael ${ }^{16}$ citan que esta patología de amplia distribución ha sido descrita ya en algunos continentes como Asia, Europa, África y América. Según Boeri y cols. ${ }^{17}$, la transmisión al hombre puede ser por contacto con el semen, orina, $\mathrm{y} / \mathrm{o}$ fetos abortados de animales infectados, lo que alerta sobre el peligro de contaminar el medioambiente.

La Organización Mundial de la Salud (OMS) clasifica la brucelosis dentro de la categoría de enfermedades infecciosas de origen bacteriano relacionadas con salud ocupacional y enfermedades de tipo profesional de notificación obligatoria ${ }^{18}$. Las personas que tienen un mayor riesgo de contraer la patología son el personal a cargo del cuidado de cachorros en perreras y criaderos ${ }^{10}$, personal de laboratorio que ha estado estrechamente en contacto con el patógeno ${ }^{13,19}$, peluqueras caninas y médicos veterinarios ${ }^{20}$. Sin embargo, la población más susceptible son los propietarios de edad avanzada, mujeres embarazadas, pacientes inmunodeprimidos (con trasplantes, cáncer e infección por VIH $)^{13,21}$ y niños bajo 6 años de edad, quienes tienen mayor contacto con sus perros ${ }^{22}$.

Debido a la gran dificultad diagnóstica de la brucelosis, se cree que se subestiman los casos positivos, por lo que esta zoonosis sigue siendo un problema de Salud Pública, tanto en países desarrollados como en el mundo entero ${ }^{19}$, ya que según la OMS, cada año se registra medio millón de nuevos casos de brucelosis (por especies patógenas) en el mundo ${ }^{14}$. En la literatura médica se describen métodos que principalmente se enfocan al diagnóstico del género Brucella para posteriormente identificar la especie, a través de ELISA, RSAT o RPC principalmente, puesto que son las técnicas con mayor especificidad y sensibilidad ${ }^{9}$.

Al realizar un análisis mediante cultivo se pueden observar colonias con características rugosas y granulares $^{13,23} \mathrm{y}$, según el pH del medio, varía el desarrollo de las cepas. Si es un pH 7,2 crecerán con mayor facilidad cepas de tipo mucoide la cual se denomina $\mathrm{M}+$ pero, si el $\mathrm{pH}$ es de 6,5 el cultivo favorecerá el desarrollo de cepas no mucoides o $\mathrm{M}^{24}$. Según lo descrito en el Center for Food Security and Public Health, esta última cepa es menos virulenta $^{11}$, puesto que ocasiona una respuesta inmune débil ${ }^{24,25}$. Otra característica de la cepa M-, es que se utiliza como antígeno en pruebas serológicas ${ }^{11}$. Esta cepa, que se creía inofensiva para el ser humano, en la actualidad se reconoce que también es capaz de producir enfermedad igual que la cepa $\mathrm{M}+25$. Se han reportado casos que revelan que el personal de laboratorio es quien tiene mayor riesgo de exposición a la cepa M-, puesto que es utilizada como antígeno para las pruebas serológicas ${ }^{11}$. El período de incubación se ha estimado que va de 2 a 4 semanas, pero eventualmente puede ser de hasta 6 semanas $^{12}$.

En la actualidad, investigaciones realizadas en perros domésticos que viven en zonas urbanas de nuestro país, entregaron datos de prevalencia de $11,25 \%{ }^{26}$ y estudios no publicados aseguran que existe una prevalencia de 15$30 \%$ de brucelosis canina, según el Ministerio de Salud ${ }^{12}$.

Los animales seropositivos alojados en lugares de albergue masivo deben ser aislados de los animales sanos manteniendo estrictas medidas de higiene ${ }^{13}$, con el fin de evitar que éstos se contagien y de esta manera también evitar que la enfermedad pueda seguir diseminándose en el ambiente ${ }^{2,16}$. Posterior a esto, algunos autores recomiendan idealmente el sacrificio mediante la eutanasia como principal estrategia de control en perreras y criaderos $^{10,16,27}$, lo cual no se aplica en mascotas con dueños, ya que en este caso la recomendación es no utilizar los animales para la reproducción y castrar inmediatamente a los machos y hembras para luego seguir con un tratamiento antimicrobiano ${ }^{13}$.

La prevención de la enfermedad es principalmente mediante el control de la infección en perros, puesto que hasta la fecha no existe una vacuna que sea capaz de producir una inmunidad satisfactoria contra la brucelosis en personas $^{28}$. Por esto, es necesario prevenir aplicando medidas de higiene y desinfección de los lugares de albergue masivo de animales (caniles y criaderos), laboratorios y asegurando la higiene personal. Además, tanto propietarios como personas que están estrechamente ligadas a ella (por el tipo de trabajo que realizan), deben utilizar siempre la indumentaria necesaria para evitar el contagio. Asimismo deben tomar precauciones para evitar la contaminación de la piel, inhalación o ingestión accidental de estos microorganismos al manejar material contaminado, al estar presente en el parto de una perra o realizar una necropsia; esta patología se considera una enfermedad ocupacional ${ }^{11}$. Sin embargo, el principal método de prevención de esta patología a mediano plazo es establecer programas de tenencia responsable de mascotas, puesto que mediante la educación se pueden obtener buenos resultados.

Estudios realizados en Chile revelan que la calidad de vida que tienen las mascotas está directamente relacionada con el nivel educacional y económico de sus propietarios, ya que esto posibilita la mantención y tenencia responsable. Sin embargo, en forma independiente del estrato socio-económico en que se encuentren los propietarios, es necesario crear conciencia en toda la población, ya sean de altos o bajos recursos, con el fin de prevenir esta y otras 
zoonosis a mediano plazo, pues es el dueño quien tiene que proporcionar cuidados básicos a su mascota, ya que la protección y cuidado de éstos, redunda en una mejor salud pública y ambiental ${ }^{29}$.

Teniendo en cuenta la falta de datos epidemiológicos regionales y el carácter universal de esta zoonosis, el objetivo de este estudio fue determinar la prevalencia de $B$. canis en perros vagos de la ciudad de Temuco albergados en el Canil Temuco.

\section{Material y Métodos}

Este estudio, financiado por el proyecto FONIS SA10I20029, contó con el auspicio y asociatividad entre la SEREMI de Salud Araucanía Sur, Municipalidad de Temuco y Universidad Católica de Temuco.

El presente estudio, de carácter cuantitativo, corresponde a un diseño de corte transversal de la zoonosis en perros vagos capturados en la ciudad de Temuco, durante el 2011 y alojados en el Canil Temuco, que se encuentra bajo la administración de la Escuela de Medicina Veterinaria de la Universidad Católica de Temuco.

Este proyecto se desarrolló en un período de 18 meses, se realizó por un método no probabilístico intencionado y se seleccionaron los animales que cumplían los criterios de haber sido ingresados como máximo durante las $48 \mathrm{~h}$ previas a la toma de muestra y ser considerados vagos. Estos perros, capturados por personal de la Municipalidad de Temuco, deambulaban libremente por las calles de la ciudad, plazas, campamentos y el Vertedero Municipal, sin tipo alguno de restricción. Además se encontraban en estrecho contacto con otros canes vagos y posiblemente también con animales domésticos de hábitos callejeros.

Para el cálculo del tamaño de la muestra se empleó la fórmula sugerida por Thrusfield ${ }^{30}$ :

$$
\mathrm{n}=1,96^{2} \mathrm{P}_{\exp }\left(1-\mathrm{P}_{\exp }\right) / \mathrm{d}^{2}
$$

La prevalencia estimada fue fijada en $50 \%$, ya que se desconoce la prevalencia de la zoonosis, estableciendo un IC de $95 \%$, con lo que se obtuvo un tamaño de muestra de 384 perros. Sin embargo, el número de perros vagos establecido fue de 400 a los efectos de salvaguardar las posibles exclusiones según los criterios fijados.

El procedimiento de toma de muestras e identificación de agentes infecciosos se realizó bajo medidas de seguridad física, bioseguridad, medidas higiénicas y bajo las normas de bienestar animal. Las muestras fueron obtenidas mediante punción de la vena cefálica antebraquial, quedando como segunda opción la vena safena. Una vez extraídas las muestras mediante un tubo Vacutainer $^{\mathbb{B}}$ estéril sin anticoagulante, se rotularon con el número de identificación del canino y se trasladaron refrigeradas al laboratorio, para luego de su coagulación ser centrifugadas por $5 \mathrm{~min}$ a $3.500 \mathrm{rpm}$. Los sueros obtenidos se congelaron a $-20^{\circ} \mathrm{C}$ hasta el momento de su análisis.

Para la identificación de animales positivos a B. canis se utilizó la técnica de inmunocromatografía (IC), utili-

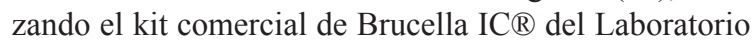
Biopronix-Italia. Sobre la membrana de nitrocelulosa, a nivel de la ventana $T$, se encuentra adherido el antígeno (Ag) específico de B. canis, mientras que a nivel de la ventana $\mathrm{C}$ se ubica una proteína específica para la verificación del ensayo. La muestra añadida a la ventana $\mathrm{S}$ migra hacia la ventana $\mathrm{T}$, donde las inmunoglobulinas específicas de B. canis se unirán al Ag adherido a la membrana de nitrocelulosa. La ausencia de la línea en la ventana $\mathrm{T}$ indicará un resultado negativo. El reactivo en la ventana $\mathrm{C}$ se unirá al anticuerpo conjugado con oro coloidal, tanto en las muestras positivas como en las negativas. La línea en la ventana $\mathrm{C}$ garantiza que el ensayo ha sido realizado correctamente ${ }^{31}$.

Este inmunoensayo cromatográfico tiene una sensibilidad y especificidad de 95,8 y 99,7\% respectivamente, lo cual es comparable con los métodos serológicos y bacteriológicos convencionales, considerándose una herramienta útil, rápida y precisa para la detección cualitativa de anticuerpos de $B$. canis $^{32}$. Es capaz de detectar anticuerpos en sangre a partir de la tercera semana post infección y, en algunos casos, a partir de la segunda semana $^{33}$.

Estadística. El análisis estadístico de los resultados de las muestras a $B$. canis se realizó mediante el programa estadístico SPSS, aplicando estadística descriptiva y $\chi^{2}$ para establecer si los animales positivos presentaban independencia de la edad y el sexo, a un nivel de significación de $5 \%$.

\section{Resultados}

Se obtuvo muestra en un total de 400 caninos, de los cuales 228 (57\%) de las muestras analizadas correspondieron a hembras y 172 eran machos.

El rango de edad de los animales estudiados fue de 2 meses a 12 años, con una mediana de 3 años. Los caninos mayores de un año de edad representaban el $87,7 \%(351 / 400)$.

De las 400 muestras analizadas con el método de inmunocromatografía, en cuatro se encontraron anticuerpos contra $B$. canis, lo cual representó una seroprevalencia de $1 \%(4 / 400)$ de esta bacteria en perros vagos de la ciudad de Temuco (Figura 1, Tabla 1). De ellos, una mitad (0,5\%) eran machos. (Figura 2).

Con respecto a los grupos etarios, las cuatro muestras que resultaron positivas (1\%) correspondieron a animales mayores de 1 año (Figura 3). 
Figura 1. Representación gráfica de la distribución porcentual de animales positivos y negativos a $B$. canis.

Figura 2. Representación gráfica de recuento de presentación de $B$. canis según sexo.

Figura 3. Representación gráfica de frecuencia de presentación de $B$. canis según edad.
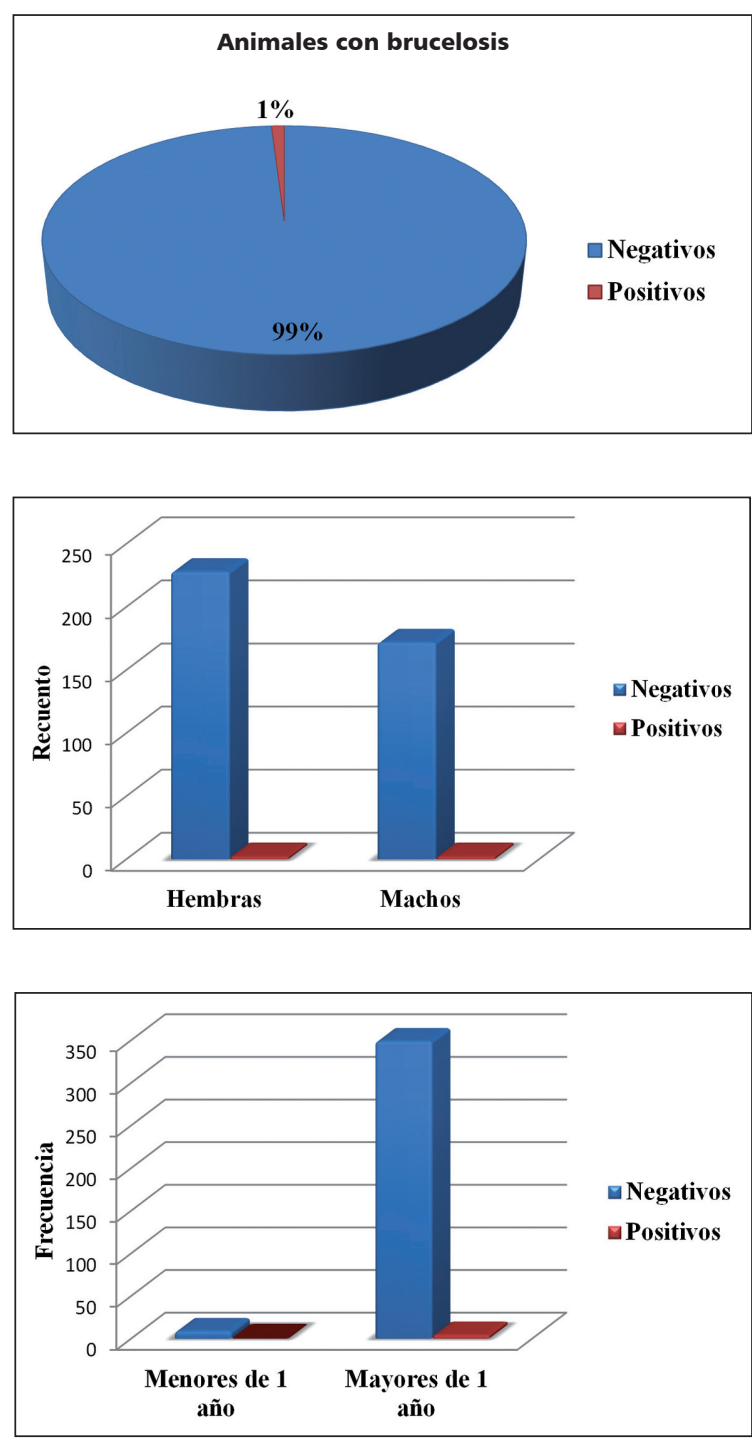

\section{Discusión}

La seropositividad de $B$. canis revelada en este estudio (1\%) es similar a lo descrito por Reis y cols. ${ }^{34}$, en 500 perros vagos de la ciudad de São João da Boa Vista del Estado de São Paulo, entre los años 2002 y 2003, quienes determinaron una prevalencia de $0,8 \%$. Asimismo, en Villavicencio, Colombia, la seroprevalencia en perros fue de 1,49\% (3 de 201) durante el año 2009; dos eran domésticos $(2 / 101)$ y uno vago $(1 / 100)^{35}$. Por otra parte, en una investigación en perros con dueños de la micro-región de Botucatu, São Paulo, se demostró que sólo 1,77\% de 1.072 perros fueron positivos a $B$. canis $^{36}$; esta seroprevalencia es menor comparada con otros estudios realizados en perros vagabundos o de refugio. Por ejemplo, en el año 2008, en Medellín, Colombia se estudió a 221 perros de
Tabla 1. Animales seropositivos para Brucella canis

\begin{tabular}{|lcc|} 
& Frecuencia & Porcentaje válido \\
Negativos & 396 & 99,0 \\
Positivos & 4 & 1,0 \\
Total & 400 & 100,0 \\
\hline
\end{tabular}

los cuales 6,78\% fueron positivos ${ }^{23}$. Asimismo, en Buenos Aires, Argentina, encontraron 7,3\% de positividad, en un total de 219 perros $^{17}$; en Turquía hubo un mayor número de reactores (28\%) en un estudio sobre 362 perros $^{37}$. Asimismo en Colombia se obtuvo una seropositividad de $11 \%{ }^{38}$ y en Alfenas, Brasil, en el año 2004 se describió una positividad de $14,2 \%$ entre 635 perros analizados ${ }^{39}$.

Se aprecia una falta de uniformidad en los criterios de inclusión de las poblaciones estudiadas en las diferentes investigaciones mencionadas; en muchos casos no se discriminó entre mascotas, perros callejeros y perros vagos, incluyendo algunos estudios a animales con signos sugerentes de brucelosis canina, donde los resultados arrojaron cifras mayores. Un claro ejemplo de esto es un estudio efectuado en Turquía donde $B$. canis fue aislada de cuatro linfonodos inguinales de un total de 48 muestras $(8,3 \%)^{40}$ y en Buenos Aires se aisló en $14,7 \%$ de los casos $(33 / 224)^{41}$. Ahora bien, en un estudio llevado a cabo en 171 perros de criaderos en São Paulo, donde al menos $22,8 \%$ tenía signos compatibles con la patología, 33,91\% de ellos fueron seropositivos ${ }^{42}$.

Fuentes bibliográficas indican que la mayor prevalencia se describe claramente en los criaderos, quedando en evidencia la importancia de la contaminación ambiental en la persistencia de la enfermedad. Los autores que testearon exclusivamente animales de criaderos o con antecedentes de alteraciones reproductivas (como abortos, muerte neonatal, orquitis, entre otros) y que mantenían contacto entre animales enfermos y sanos, obtuvieron las más altas prevalencias. Un claro ejemplo de esto es un estudio realizado en un criadero canino en Irán, con historia de afecciones reproductivas, donde se analizaron 21 muestras, de las cuales $57,2 \%$ fueron positivas ${ }^{43}$. En las mismas circunstancias, en el Distrito Federal de México, en el año 2004 se analizaron 56 muestras de perros machos, de las cuales 24 fueron positivas $(42,8 \%)^{44}$. Por su parte, Megid y cols. ${ }^{45}$ obtuvieron $57,1 \%$ de positividad en 151 perros Poodle de criadero. Vargas y cols. ${ }^{46}$, también encontraron altos valores $(72,7 \%), 8$ positivos en un total de 11 caninos estudiados.

Los caninos machos acostumbran a marcar y olfatear áreas orinadas por otros perros y, como bien se sabe, la orina es el principal medio de diseminación de las brucelas; esta bacteria ${ }^{13} \mathrm{es}$, además, capaz de mantenerse viable en el medio ambiente debido a sus propiedades que 
le permiten sobrevivir en climas húmedos ${ }^{47}$, tal como es en nuestra región en épocas de otoño e invierno, todo lo cual justifica los cuatro casos positivos encontrados en agosto-septiembre.

Otro factor de variabilidad en los resultados es la capacidad de diagnosticar la enfermedad. El método utilizado en este estudio fue un ensayo inmunocromatográfico, que detecta, como ya se ha señalado, anticuerpos en sangre a partir de la tercera semana post infección y, en algunos casos, incluso a partir de la segunda semana. Posee además un alto índice de sensibilidad (96\%) y especificidad $(100 \%)^{33,43}$; sin embargo, según lo descrito por varios autores, el diagnóstico definitivo de $B$. canis requiere la aplicación simultánea de diversas técnicas de laboratorio, debido a la falta de un método diagnóstico único y fiable $\mathrm{e}^{48,49}$, lo cual es primordial, ya que un mal diagnóstico es sinónimo de un importante número de casos que pueden seguir siendo "falsos negativos". Otra posible causa de bajos resultados positivos es una supuesta resistencia por parte de los perros callejeros a $B$. canis, pero esto no se ha comprobado aún según Ruiz y cols. ${ }^{23}$. Otra herramienta muy útil para el diagnóstico es la RPC, técnica molecular muy rápida que detecta $\mathrm{ADN}$ y se puede utilizar en sangre total, leche, secreción vaginal, semen y linfonodos, lo que aumenta su posibilidad de uso ${ }^{25,50}$. Se puede aplicar en todas las fases de la infección, haciéndola muy útil en animales crónicos serológicamente negativos ${ }^{25,51}$; además no es una herramienta peligrosa para el personal de laboratorio lo que aumenta sus ventajas de uso $^{50}$.

La gran mayoría de los perros analizados en este estudio eran mestizos, los que tienen un mayor riesgo de infección que los animales de raza $^{52}$, pues la gran mayoría eran vagos ${ }^{13}$. Por otra parte, todos los animales positivos de este estudio eran mayores de un año, concordando con otros investigadores, lo que se justifica debido que a esta edad se encuentran maduros sexualmente ${ }^{39}$. Esto permite una mayor probabilidad de contacto genital con animales infectados, pues esta es la principal vía de diseminación entre los animales ${ }^{34,35}$. No existe, sin embargo, realmente una asociación significativa entre las variables sexo y edad, lo que permite establecer que estos no son factores predisponentes de $B$. canis $^{23}$.

Aunque esta investigación no pretendió investigar la ocurrencia de casos clínicos humanos asociados a la infección canina, son muchos los estudios que demuestran el contagio de $B$. canis en personas convivientes con caninos infectados ${ }^{53,22}$, probablemente por el estrecho contacto que mantiene el hombre con sus mascotas, lo cual resulta riesgoso para su salud ${ }^{54}$. Los datos del presente trabajo alertan sobre el peligro potencial existente tanto para los animales, como para las personas y el medio ambiente ${ }^{55}$, he ahí su importancia. Por consiguiente, aunque exista una baja prevalencia de esta bacteria, el riesgo está presente, ya que al ser perros vagos, es alta la probabilidad de diseminación del agente patógeno ${ }^{34}$. Además, la presencia de $B$. canis en un pequeño porcentaje en caninos es suficiente para considerarlo un problema de importancia epidemiológica ${ }^{27}$. Los resultados de este estudio son una advertencia para los profesionales del área de la Salud Animal y para los de Salud Pública, quienes deben considerar la presencia de $B$. canis en nuestro país y con esto adoptar medidas de control, prevención y mitigación, ya que la enfermedad en mascotas es fácilmente controlable, pero en la población de perros vagos es más complejo. Como consecuencia del potencial zoonótico emergente de esta enfermedad, debiese ser evaluada la necesidad de coordinar sistemas de vigilancia de brucelosis canina en nuestro país ${ }^{22}$.

Los cambios en el entorno urbano debido a la expansión de comunidades marginales ha dado lugar a un aumento de las poblaciones de perros callejeros y vagos en el entorno peridomicilio. La clave para reducir la transmisión de enfermedades infecciosas en la Salud Pública, es que todos tengan un acceso equitativo a los servicios de saneamiento?.

En Chile, desde la descripción de la enfermedad en el año 1978, son pocos los estudios realizados para determinar su prevalencia. Estos podrían ser de gran aporte, pues permitirían conocer la situación real de esta zoonosis. En la comuna de San Bernardo, Región Metropolitana, se realizó un estudio en 80 animales domésticos con los métodos de IFI e IC; de éstos, 9 resultaron positivos con IFI, encontrándose una prevalencia de $11,25 \%{ }^{26}$. El año recién pasado, en una circular de vigilancia epidemiológica del Ministerio de Salud se mencionó que en estudios no publicados de perros que viven en zonas urbanas del país, existe una prevalencia de $15-30 \%$ de B. canis $^{12}$. Por el contrario, los datos encontrados sobre zoonosis por $B$. canis son escasos. El más actualizado es un estudio hecho en Santiago donde se analizaron por serología 100 personas (médicos veterinarios y peluqueras caninas) donde 6 casos asintomáticos fueron positivos ${ }^{20}$, lo que demuestra la poca vigilancia epidemiológica de esta zoonosis sobre todo en los seres humanos, ya que las notificaciones reportadas son bajas.

En conclusión, la brucelosis canina debe ser considerada como un problema de Salud Pública y Ambiental. Si bien la tasa detectada en nuestro estudio es baja, requiere de más investigaciones para comprender mejor su epidemiología, estrategias de control y prevención evitando así un incremento en su prevalencia y disminuyendo de esta manera el riesgo en la población humana.

\section{Resumen}

Introducción: Brucella canis es responsable de la brucelosis en perros, provocándoles trastornos reproductivos y es considerada una zoonosis, ya descrita en 
varios países. Los datos epidemiológicos en nuestro medio son exiguos. Objetivo: Determinar la prevalencia de $B$. canis en perros vagos capturados en la ciudad de Temuco y albergados en el Canil Temuco. Material y Métodos: Estudio de tipo cuantitativo y de corte transversal. Se utilizaron 400 muestras de perros de ambos sexos, diferentes edades y principalmente mestizos, procesadas mediante la prueba de inmunocromatografía. Resultados:
Se detectaron anticuerpos anti- $B$. canis en 4 muestras lo cual representó $1 \%$ de la población estudiada, 2 hembras $(0,5 \%)$ y 2 machos $(0,5 \%)$. Conclusión: El hallazgo de perros serológicamente positivos a $B$. canis, es baja pero no deja de ser un indicador del riesgo en el que se encuentra la salud de la población humana si no se mantiene un adecuado control sanitario de las mascotas, como ocurre con los perros vagos.

\section{Referencias bibliográficas}

1.- Vega C, Ariza R, Rodríguez F. Brucelosis. Una infección vigente. Acta Médica Grupo Ángeles 2008; 6 (4): 158-65.

2.- Seleem M, Boyle S, Sriranganathan M. Brucellosis: A re-emerging zoonosis. Vet Microbiol 2010; 140: 392-8.

3.- Kathleen M, Lynn T. Brucellosis. JAVMA 2008; 233 (6): 900-8.

4.- Scholz H, Hubalek Z, Sedlacek I, Vergnaud G, Tomaso H, Dahouk S, et al. Brucella microti sp. nov., isolated from the common vole Microtus arvalis. Int J Syst Evol Microbiol 2008; 58: 375-82.

5.- Foster G, Osterman B, Godfroid J, Jacques I, Cloeckaert A. Brucella ceti sp. nov. and Brucella pinnipedialis sp. nov. for Brucella strains with cetaceans and seals as their preferred hosts. Int J Syst Evol Microbiol 2007; 57: 2688-93.

6.- Acha N, Szyfres B. Zoonosis y enfermedades transmisibles comunes al hombre y a los animales. Tercera ed. Washington: Editorial PAHO. 2003.

7.- Rousseau P. Brucella canis infection in a woman with fever of unknown origin. Postgrad Med 1985; 78: 249: 253-4, 7.

8.- $\quad$ Ying W, Nguyen M Q, Jahre J A. Brucella canis endocarditis: case report. Clin Infect Dis 1999; 29: 1593-4.

9.- Marzetti S, Carranza C, Roncallo M, Escobar G I, Lucero NE. Recent trends in human Brucella canis infection. Comp Immunol Microbiol Infect Dis 2013; 36 (1): 55-61. Epub 2012 oct 4.

10.- Hollett RB. Canine brucellosis: Outbreaks and compliance. Theriogenology 2006; 66: 575-87.

11.- Centers for Disease Control and Prevention and Public Health. Brucelosis. Disponible en: http:// www.cfsph.iastate.edu/Factsheets/es/brucelosis. pdf [Acceso el 16 de marzo de 2012].

12.- Ministerio de Salud. Brucelosis. 2011. Circular $\mathrm{N}^{\circ} \mathrm{B} 51 / 03$. Departamento de Epidemiología. Disponible en: http://epi.minsal.cl/epi/html/ normas/circul/Circular_BRUCELOSIS.pdf [Acceso el 7 de agosto de 2011].

13.- Wanke M. Canine brucellosis. An Reprod Sci 2004; 82-83: 195-207.

14.- Nimri LF. Diagnosis of recent and relapsed cases of human brucellosis by PCR assay. BMC Infect Dis 2003; 28: 3-5.

15.- Lucero N, Jacob N, Ayala S, Escobar G, Tuccillos P, Jacques I. Unusual clinical presentation of brucellosis caused by Brucella canis. J Med Microbiol 2005; 54: 505-8.

16.- Shin J, Carmichael E. Canine brucelosis caused by Brucella canis. Disponible en: http://www. ivis.org/advances/infect_dis_carmichael/shin/ ivis.pdf [Acceso el 12 de marzo de 2011].

17.- Boeri E, Escobar G I, Ayala S M, Sosa-Estani S, Lucero N E. Canine brucellosis in dogs in the city of Buenos Aires. Medicina (B. Aires) 2008; 68 (4): 291-7.

18.- Ramírez H, Calle S, Echevarria L, Morales S. Prevalencia de brucelosis canina en dos distritos de la Provincia constitucional del Callao. Rev Inv Vet Perú 2006; 17 (1): 39-43.

19.- Zinsstag J, Schelling E, Roth F, Bonfoh B, de Savigny D, Tanner M. Human benefits of animal interventions for zoonosis control. Emerg Infect Dis 2007; 13 (4): 527-31.

20.- Valenzuela N, García P, Salgado S, Concha M, Abarca K, López J, et al. Seroprevalencia en humanos de Brucella canis en un grupo con exposición ocupacional. Libro de resúmenes XXII Congreso Chileno de Infectología, Puerto Varas 26 al 29 de octubre, 2005. Resumen: $\mathrm{CO} 37$.

21.- Abarca V K, López del P J, Peña D A, López J C. Tenencia y estado de salud de mascotas de niños inmunocomprometidos, con énfasis en enfermedades zoonóticas. Rev Chilena Infectol 2011; 28 (3): 205-10.

22.- Lucero N E, Corazza R, Almuzara M N, Reynes E, Escobar G I, Boeri, E, et al. Human Brucella canis outbreak linked to infection in dogs. Epidemiol Infect 2010; 138 (2): 280-5.

23.- Ruiz J, Giraldo C, López L, Chica J. Seroprevalencia de Brucella canis en perros callejeros del Centro de Bienestar Animal "La Perla", Medellín (Colombia), 2008. Rev Colomb Cienc Pecu 2010; 23: 166-72.

24.- Ardoino M, Baruta A, Toso E. Brucelosis canina. Cien Vet 2006; 8 (1): 49-60.

25.- Wallach J C, Giambartolomei G H, Baldi P C, Fossati C A. Human Infection with M- strain of Brucella canis. Emerg Infect Dis 2004; 10 (1): 146-8.

26.- López J, Obrist W, García P, Valenzuela N,
Guerrero J. Seroprevalencia de Brucella canis en una población canina perteneciente a la comuna de San Bernardo, Región Metropolitana. Libro de Resúmenes XXII Congreso Chileno de Infectología, Puerto Varas, 26 al 29 de octubre de 2005. Resumen: P17.

27.- Brennan S J, Ngeleka M, Philibert H M, Forbes L B, Allen AL. Canine brucellosis in a Saskatchewan kennel. Can Vet J 2008; 49 (7): 703-8.

28.- Corbel M. Brucellosis: an overview. En: Emerging Infectious Diseases, 1st International Conference on Emerging Zoonoses, Jerusalem, abril-junio, 1997. Israel. Emerg Infect Dis 1997; 3 (2): 213-21.

29.- Herrera S. 2007 Tenencia responsable de mascotas caninas en la comuna de Providencia. Región Metropolitana. Universidad Iberoamericana de Ciencias y Tecnología. Disponible en http://www.iberovet.cl/tesis/ images/pdf/42.pdf

30.- Thrusfield, M. Veterinary Epidemiology. 2nd Ed. Oxford: Blackwell Science Ltda. 1995.

31.- Agrolabo S.p.A 2011. Test principle Brucella IC. Disponible en http://www.agrolabo.it/EN/ index.php?page $=$ articolo $\&$ menu $=11 \& \mathrm{ID}=47$ $\&$ ID_prod $=10 \&$ om $=[$ Acceso el 4 de junio de 2012].

32.- Mosallanejad B, Najafabadi M, Avizeh R, Mohammadian N. A serological survey on Brucella canis in companion dogs in Ahvaz. IJVR 2009; 10 (4): 383-6.

33.- Kim J, Lee W, Han M, Bae D, Jung S, Oh J, et al. Evaluation of immunochromatographic assay for serodiagnosis of Brucella canis. J Vet Med Sci 2007; 69 (11): 1103-7.

34.- Reis C, Hoffman R, Santos R, Turri R, Oriani M. Pesquisa de anticorpos anti-Brucella canis e anti- Brucella abortus em cães errantes da cidade de São João da Boa Vista, Estado de São Paulo, Brasil (2002-2003). Braz J Vet Res Anim Sci, São Paulo 2008; 45: 32-4.

35.- Pardo A, Pérez C, Góngora A, Gómez L, Moreno A. Encuesta exploratoria de infección por Brucella canis en perros de VillavicencioColombia. Rev MVZ Córdoba 2009; 14 (2): 1690-6.

36.- Moraes C, Megid J, Souza L, Crocci A. Prevalência da brucelose canina na microrregião Da serra de botucatu, São Paulo, Brasil. Arq Inst 
Biol, São Paulo 2002; 69 (2): 7-10.

37.- Öncel T, Akan M, Sareyypoúlu B, Tel O, Ciftci A. Seroprevalence of Brucella canis infection of dogs in two provinces in Turkey. Turk J Vet Anim Sci 2005; 29: 779-83.

38.- Giraldo C, Ruiz Z, Olivera M. Brucella canis en Medellín (Colombia), un problema actual. Rev UDCA Act \& Div Cient 2009; 12 (1): 51-7

39.- Almeida A, Santorelli A, Bruzadelli R, Oliveira M. Soroepidemiologia da brucelose canina causada por Brucella canis e Brucella abortus na cidade de Alfenas, MG. Arq Bras Med Vet Zootec 2004; 56(2): 275-6.

40.- Aras Z, Uçan U. Detection of Brucella canis from inguinal lymph nodes of naturally infected dogs by PCR. Theriogenology 2010; 74 (4): 658-62.

41.- López G, Ayala S M, Efron A M, Gómez C F, Lucero N E. A serological and bacteriological survey of dogs to detect Brucella infection in Lomas de Zamora, Buenos Aires province. Rev Argent Microbiol 2009; 41 (2): 97-101.

42.- Keid B, Soares M, Morais M, Richtzenhain J, Vasconcellos A. Brucella spp. isolation from dogs from commercial breeding kennels in São Paulo state, Brazil. Braz J Microbiol 2004; 35 :
161-6.

43.- Amin M, Mogheiseh A. Outbreak investigation of brucellosis at a Kennel in Iran. Pak Vet J 2011; 31: 379-80.

44.- Briseño H, Páramo R, Flores R, Suárez F. Problemas reproductivos en perros machos infectados con Brucella canis. Vet Méx 2004; 35 (2): 121-8.

45.- Megid J, Brito A, Moraes C, Fava N, Agottani J. Epidemiological assessment of canine brucelosis. Arq Bras Med Vet Zootec 1999; 51 (5): 439-40

46.- Vargas A, Lazzari A, Dutra V, Poester F. Brucelose canina: relato de caso. Cienc Rural 1996; 26 (2): 305-8.

47.- Centers for Disease Control and Prevention and Public Health. Canine brucellosis: Brucella canis. Disponible en: http://www.cfsph.iastate. edu/Factsheets/pdfs/brucellosis_canis.pdf [Acceso el 10 de enero de 2012].

48.- Liu C, Hsu Y. The role of comparative pathology in the investigation of zoonoses. Tzu Chi Med J 2007; 19 (3): 127-33.

49.- Gyuranecz M, Szeredi L, Rónai Z, Dénes B, Dencso L, Dán A, et al. Detection of Brucella canis-induced reproductive diseases in a kennel. J Vet Diagn Invest 2011; 23 (1): 143-7.
50.- Olivera M, Giraldo C, Di-Lorenzo C. Identificación por PCR de Brucella canis en sangre y leche canina: Reporte de un caso. Arch Med Vet 2011; 43: 295-8.

51.- Corrente M, Franchini D, Decaro N, Greco G, D'Abramo M, Greco M, et al. Detection of Brucella canis in a dog in Italy. New Microbiol 2010; 33 (4): 337-41.

52.- Borie C, Sánchez M. Brucelosis en el perro. TecnoVet 2002;8(1) Disponible en: http:// www.revistas.uchile.cl/index.php/RT/article/ viewArticle/10502/10556 [Acceso el 18 de febrero de 2012].

53.- Olivera M, Di-Lorenzo C. Aislamiento de Brucella canis en un humano conviviente con caninos infectados. Informe de un caso. Colomb Med 2009; 40 (2): 218-20.

54.- Brower A, Okwumabua O, Massengill C, Muenks Q, Vanderloo P, Duster M, et al. Investigation of the spread of Brucella canis via the U.S. interstate dog trade. Int J Infect Dis 2007; 11 (5): 454-8.

55.- Organización Mundial de la Salud. Brucellosis in humans and animals. Disponible en: http:// www.who.int/csr/resources/publications/ Brucellosis.pdf [Acceso el 30 de agosto de 2011]. 